\title{
A phenomenological analysis of the longitudinal structure function at small $x$ and low $Q^{2}$
}

\author{
V.P. Gonçalves ${ }^{1}$, M.V.T. Machado1,2 \\ 1 Instituto de Física e Matemática, Universidade Federal de Pelotas, Caixa Postal 354, CEP 96010-090, Pelotas, RS, Brazil \\ 2 Universidade Estadual do Rio Grande do Sul, UERGS, Engenharia de Bioprocessos e Biotecnologia, Bento Gonçalves, RS, \\ Brazil \\ 3 High Energy Physics Phenomenology Group, GFPAE, IF-UFRGS, Caixa Postal 15051, CEP 91501-970, Porto Alegre, RS, \\ Brazil
}

Received: 23 June 2004 / Revised version: 13 July 2004 /

Published online: 14 September 2004 - (C) Springer-Verlag / Società Italiana di Fisica 2004

\begin{abstract}
The longitudinal structure function in deep-inelastic scattering is one of the observables from which the gluon distribution can be unfolded. Consequently, this observable can be used to constrain the QCD dynamics at small $x$. In this work we compare the predictions of distinct QCD models with the recent experimental results for $F_{\mathrm{L}}\left(x, Q^{2}\right)$ at small $x$ and low $Q^{2}$ obtained by the $\mathrm{H} 1$ Collaboration. We focus mainly on the color dipole approach, selecting those models which include saturation effects. Such models are suitable at this kinematical region and also resum a wide class of higher-twist contributions to the observables. Therefore, we investigate the influence of these corrections to $F_{\mathrm{L}}$ in the present region of interest.
\end{abstract}

\section{Introduction}

The small $x$ regime in deep-inelastic scattering (DIS) is one of the frontiers of quantum chromodynamics (QCD). This represents the challenge of studying the interface between perturbative and non-perturbative QCD, with the characteristic feature that the transition is taken in a kinematical region where the strong coupling constant $\alpha_{\mathrm{s}}$ is small. That region has been explored by the electronproton $(e p)$ scattering at HERA, which has shown a striking rise of the proton structure function $F_{2}\left(x, Q^{2}\right)$ for values $x<10^{-2}$. This behavior implies that the cross section increases faster that logarithmically with the energy, violating the Froissart bound. Therefore, new dynamical nonlinear QCD effects associated to the unitarity corrections are expected to slow down its further growth [1-7]. The search of signatures for these effects has been an active subject of research in the last years [8-24].

In particular, it has been observed that the HERA data at small $x$ and low $Q^{2}$ can be successfully described with the help of saturation models [10-12,21]. Moreover, the experimental results for the total cross section [14] and also for the inclusive charm production [20] present the property of geometric scaling, which is one of the main characteristics of the high density QCD approaches (for a recent review see e.g. [25]). The saturation (non-linear QCD) approaches are characterized by a typical scale, denoted the saturation scale $Q_{\mathrm{s}}^{2}(x)$, which is energy dependent, and marks the transition between the linear (leading twist) perturbative QCD regime and saturation domain.
As current phenomenological saturation models have indicated that for the HERA domain the saturation scale is smaller than $2 \mathrm{GeV}^{2}$, we expect that the signatures of the saturation effects become more evident in the region of small $x$ and very low $Q^{2}$. Furthermore, some of these approaches contain information of all orders in $1 / Q^{2}$, namely they resum higher-twist contributions $[26,27]$. These corrections should be important at the low $Q^{2}$ region, where the leading-twist (DGLAP) approaches would be in the limit of their applicability. Therefore, saturation models are quite suitable for the present phenomenological study of the longitudinal structure function at low $Q^{2}$.

In this work we analyze the behavior of the longitudinal structure function $F_{\mathrm{L}}\left(x, Q^{2}\right)$ in this kinematical regime. One considers several QCD theoretical approaches, focusing mainly on the saturation models. The predictions are compared with the recent (preliminary) $F_{\mathrm{L}}$ experimental results, as determined from the 1999 minimum bias and the 2000 shifted vertex H1 data [28]. A comment related to these results is in order here. The experimental determination of $F_{\mathrm{L}}$ is difficult since it usually requires cross sections measurements at different values of center of mass energy, implying a change of beam energies. An alternative possibility is to apply the radiation of a hard photon by the incoming electron. Such hard radiation results in an effective reduction of the center of mass energy. Several studies on the use of such events to measure $F_{\mathrm{L}}$ have been carried out [29]. With these measurements, which in principle could be performed in the 
near future, it may be possible to explore the structure of $F_{\mathrm{L}}\left(x, Q^{2}\right)$ in the low $x$ range. However, currently, to obtain the $F_{\mathrm{L}}$ data, the $\mathrm{H} 1$ Collaboration has parametrized the structure function $F_{2}$ taking only data for $y<0.35$, where the contribution of $F_{\mathrm{L}}$ is small. This parameterization was evolved in $Q^{2}$ according to the DGLAP evolution equations, providing predictions for $F_{2}$ in the high $y$ region which allowed, by subtraction of the contribution of $F_{2}$ to the cross section, the determination of the longitudinal structure function (for more detailed discussions see $[30,28])$. Therefore, the $F_{\mathrm{L}}$ data only are obtained after the use of a procedure in the measurements of the total cross section.

This paper is organized as follows. In the next section, we briefly review the theoretical description of $F_{\mathrm{L}}$ in the linear DGLAP (leading-twist) approximation and summarize the main expressions considering the color dipole approach. For the latter, we introduce two representative saturation models which have their phenomenological parameters well constrained by the current small $x$ experimental data. The comparison of the numerical results, further discussions and conclusions are presented in the last section.

\section{Theoretical description of $F_{\mathrm{L}}$ at small $x$}

The longitudinal structure function $F_{\mathrm{L}}$ corresponds to the interaction of the longitudinally polarized virtual photon in the one-photon-exchange mechanism of lepton-nucleon scattering. It is a very interesting dynamical quantity since, at least at low $x$, its dominant contribution comes from gluons. While in the naive parton model this structure function vanishes, at leading order in $\alpha_{\mathrm{S}}\left(Q^{2}\right)$ it acquires a leading twist contribution. At small $x$ this contribution is driven by the gluon through the $g \rightarrow q \bar{q}$ transition and, in fact, $F_{\mathrm{L}}$ can be used as a very useful quantity for a direct measurement of the gluon distribution in a nucleon. One can write the longitudinal structure function $F_{\mathrm{L}}$ in terms of the cross section for the absorption of longitudinally polarized photons as

$$
\begin{aligned}
F_{\mathrm{L}}\left(x, Q^{2}\right) & =\frac{Q^{2}(1-x)}{4 \pi^{2} \alpha_{\mathrm{em}}} \sigma_{\mathrm{L}}^{\gamma^{*} p}\left(x, Q^{2}\right) \\
& \approx \frac{Q^{2}}{4 \pi^{2} \alpha_{\mathrm{em}}} \sigma_{\mathrm{L}}^{\gamma^{*} p}\left(x, Q^{2}\right)
\end{aligned}
$$

at small $x$. Therefore, accurate measurements of $F_{\mathrm{L}}$ at low $x$ and/or $Q^{2}$ would be helpful to constrain the physics in that kinematical region. In particular, we expect that this observable may discriminate between the leadingtwist predictions, which consider the collinear factorization and parton distributions determined from global fits, and the predictions from the saturation models which resum a class of higher-twist contributions at small $x$.

While the longitudinal structure function is (at least theoretically) fairly well understood at high $Q^{2}$, very little (if anything) is known about its possible extrapolation towards the region of low $Q^{2}$ and small $x$ [31] (for recent discussions see e.g. $[32,33])$. Theoretically, we have that in the limit $Q^{2} \rightarrow 0$ the structure function $F_{\mathrm{L}}$ has to vanish as $Q^{4}$. This reflects the simple physical fact that the total cross section $\sigma_{\mathrm{L}} \approx F_{\mathrm{L}} / Q^{2}$ describing the interaction of longitudinally polarized virtual photons has to vanish in the real photoproduction limit. On the other hand, the leading-twist DGLAP MRST [34] and CTEQ [35] global fits require the gluon distribution to be valence-like or negative at small $x$ and low $Q^{2}$ in order to describe the experimental data, leading to $F_{\mathrm{L}}$ being negative at the smallest $x-Q^{2}$. At that region, a comparison of the predictions at LO, NLO and NNLO using MRST partons has shown a poor description of the experimental results [36]. However, the description is improved if a $\ln (1 / x)$ resummation is considered [37]. Here, it is important to emphasize that higher-twist contributions are not considered in these analyses. Nevertheless, in the global fit of the existing light-targets DIS data at LO, NLO and NNLO QCD approximations, Alekhin [38] has estimated the high-twist contributions to the structure functions. It was verified that these terms do not vanish up to NNLO (see Fig. 12 in that reference) and give important contributions at both small and large $x$ regions. Although the expectation that a higher twist plays an important role at very large $x$ is not new, the contribution of these terms in the small $x$ region has been a subject of discussion only in the last years. For instance, in [39] a simple parameterization of the higher-twist contribution to the $F_{2}$ structure function has been used, and it was found that for $x<0.5$ the resulting correction is small and negative but beyond 0.6 large and positive. In particular, the higher-twist contributions for $x<0.01$ are very small at the HERA low $x$ domain. In other words, the experimental results for the $F_{2}$ structure function in principle can be described by a leading-twist approximation. However, this feature can also be explained as being due to the almost complete cancellation of the twist- 4 corrections to the transverse and longitudinal structure functions [26]. Therefore, only a direct analysis of $F_{\mathrm{L}}$ could discriminate between leadingtwist and higher-twist resummations.

In order to address these issues, in what follows we present representative theoretical approaches taking into account the usual DGLAP leading twist approximation and the twist resummation rendered by the saturation models. At leading order, twist- 2 and in the infinite momentum frame the longitudinal structure function can be expressed in terms of the Altarelli-Martinelli equation [40]

$$
\begin{aligned}
& F_{\mathrm{L}}\left(x, Q^{2}\right)=\frac{\alpha_{\mathrm{s}}\left(Q^{2}\right)}{2 \pi} x^{2} \int_{x}^{1} \frac{\mathrm{d} y}{y^{3}} \\
& \quad \times\left[\frac{8}{3} F_{2}\left(y, Q^{2}\right)+4 \sum_{q} e_{q}^{2}\left(1-\frac{x}{y}\right) y g\left(y, Q^{2}\right)\right],
\end{aligned}
$$

which shows the dependence of $F_{\mathrm{L}}$ on the strong constant coupling and on the gluon density. At small $x$, the second term is the dominant one since it is driven by the gluon distribution. Consequently, (2) can be reasonably approximated by $F_{\mathrm{L}} \approx 0.3 \frac{4 \alpha_{\mathrm{s}}}{3 \pi} x g\left(2.5 x, Q^{2}\right)$ [41]. This relation demonstrates the close relation between the longitudinal structure function and the gluon distri- 
bution. In our further numerical calculations using the Altarelli-Martinelli equation, one considers as input the MRST2001(LO) [34] and GRV98LO [42] parton distributions. We use the GRV98 parameterization in order to compare the collinear approach with the experimental results for $Q^{2} \leq 1 \mathrm{GeV}^{2}$.

In the proton rest frame, the DIS process can be seen as a succession in time of three factorizable subprocesses:

(i) the photon fluctuates in a quark-antiquark pair with transverse separation $r_{\perp} \sim 1 / Q$ long after the interaction,

(ii) this color dipole interacts with the proton target,

(iii) the quark pair annihilates in a virtual photon.

The interaction $\gamma^{*} p$ is further factorized in the simple formulation [43],

$$
\sigma_{\mathrm{L}, \mathrm{T}}^{\gamma^{*} p}\left(x, Q^{2}\right)=\int \mathrm{d} z \mathrm{~d}^{2} r_{\perp}\left|\Psi_{\mathrm{L}, \mathrm{T}}\left(z, r_{\perp}, Q^{2}\right)\right|^{2} \sigma_{\mathrm{dip}}\left(x, r_{\perp}\right)
$$

where $z$ is the longitudinal momentum fraction of the quark, and $x \simeq Q^{2} / W_{\gamma p}^{2}$ is equivalent to the Bjorken variable. The photon wavefunctions $\Psi_{\mathrm{L}, \mathrm{T}}$ are determined from light cone perturbation theory and read

$$
\begin{aligned}
\left|\Psi_{\mathrm{T}}\right|^{2}= & \frac{6 \alpha_{\mathrm{em}}}{4 \pi^{2}} \sum_{f} e_{f}^{2}\left\{\left[z^{2}+(1-z)^{2}\right] \varepsilon^{2} K_{1}^{2}\left(\varepsilon r_{\perp}\right)\right. \\
& \left.+m_{f}^{2} K_{0}^{2}\left(\varepsilon r_{\perp}\right)\right\}, \\
\left|\Psi_{\mathrm{L}}\right|^{2}= & \frac{6 \alpha_{\mathrm{em}}}{\pi^{2}} \sum_{f} e_{f}^{2}\left\{Q^{2} z^{2}(1-z)^{2} K_{0}^{2}\left(\varepsilon r_{\perp}\right)\right\},
\end{aligned}
$$

where the auxiliary variable $\varepsilon^{2}=z(1-z) Q^{2}+m_{f}^{2}$ depends on the quark mass, $m_{f}$. The $K_{0,1}$ are the McDonald functions and the summation is performed over the quark flavors.

The dipole hadron cross section $\sigma_{\text {dip }}$ contains all information about the target and the strong interaction physics. There are several phenomenological implementations for this quantity $[9-12,19,21]$. The main feature of these approaches is to be able to match the soft (low $Q^{2}$ ) and hard (large $Q^{2}$ ) regimes in an unified way. In the present work, we follow the quite successful saturation models $[10,21]$, which interpolate between the small and large dipole configurations, providing color transparency behavior, $\sigma_{\text {dip }} \sim \boldsymbol{r}^{2}$, as $r \gg Q_{\mathrm{s}}$, and constant behavior at large dipole separations, $r<Q_{\mathrm{s}}$. It is important to emphasize that in the dipole models at small $x$, both $F_{\mathrm{L}}$ and $F_{2}$ are governed by $\sigma_{\text {dip }}$ and therefore behave similarly. In particular, $F_{\mathrm{L}}$ should go to zero when $Q^{2} \rightarrow 0$ at low $x$ in the dipole picture since $\left|\Psi_{\mathrm{L}}\right|^{2} \propto Q^{2}$. The parameters of the saturation models have been obtained from phenomenological adjustments to small $x$ HERA data. As a first model, we present the analytically simple GBW model, which resembles the main features of the Glauber-Mueller resummation. Its phenomenological application has been successful in a wide class of processes with a photon probe (DIS, diffractive DIS, deeply virtual Compton scattering, heavy-quark production, two-photon physics) $[10,12-18$,
20]. The parameterization for the dipole cross section in this model takes the eikonal-like form,

$$
\begin{aligned}
\sigma_{\text {dip }}\left(\tilde{x}, \boldsymbol{r}^{2}\right) & =\sigma_{0}\left[1-\exp \left(-\frac{Q_{\mathrm{s}}^{2}(x) \boldsymbol{r}^{2}}{4}\right)\right], \\
Q_{\mathrm{s}}^{2}(x) & =\left(\frac{x_{0}}{\tilde{x}}\right)^{\lambda} \mathrm{GeV}^{2},
\end{aligned}
$$

where the parameters were obtained from a fit to the HERA data producing $\sigma_{0}=23.03(29.12) \mathrm{mb}, \lambda=$ $0.288(0.277)$ and $x_{0}=3.04 \cdot 10^{-4}\left(0.41 \cdot 10^{-4}\right)$ for a three-flavor (four-flavor) analysis [10]. An additional parameter is the effective light-quark mass, $m_{f}=0.14 \mathrm{GeV}$, which plays the role of a regulator for the photoproduction $\left(Q^{2}=0\right)$ cross section.

An important aspect of the saturation models is that they resum a class of higher-twist contributions which should be non-negligible in the low $Q^{2}$ regime $[26,27]$. Consequently, in this kinematical region we may expect a discrimination between the twist-2 calculations, usually considered in the global fits of the experimental data, and the saturation models. Some hints of the differences between these models have been presented in $[26,27]$. In particular, the twist expansion of the GBW model has been calculated, with the different twist terms in the massless limit given by

Twist-4 $\sigma_{\mathrm{L}}^{(4)}=$
$\sigma_{0} \sum_{f} e_{f}^{2} \frac{\alpha_{\mathrm{em}}}{\pi}\left(-\frac{94}{75} \xi^{2}+\frac{4}{5} \psi(3) \xi^{2}-\frac{4}{5} \xi^{2} \ln (1 / \xi)\right)$,

Twist-6 $\quad \sigma_{\mathrm{L}}^{(6)}=$

$$
\sigma_{0} \sum_{f} e_{f}^{2} \frac{\alpha_{\mathrm{em}}}{\pi}\left(\frac{654}{1225} \xi^{3}-\frac{36}{35} \psi(4) \xi^{3}+\frac{36}{35} \xi^{3} \ln (1 / \xi)\right),
$$

Twist- $8 \quad \sigma_{\mathrm{L}}^{(8)}=$

$$
\begin{aligned}
\sigma_{0} \sum_{f} & e_{f}^{2} \frac{\alpha_{\mathrm{em}}}{\pi}\left(-\frac{1636}{18375} \xi^{4}+\frac{48}{175} \psi(5) \xi^{4}\right. \\
- & \left.\frac{48}{175} \xi^{4} \ln (1 / \xi)\right)
\end{aligned}
$$

where $\xi=\frac{Q_{\mathrm{s}}^{2}}{Q^{2}}$ is the scaling variable which appears in the geometric scaling property of the inclusive cross section and $\psi(x)$ is the digamma function. The results above can be contrasted to the leading-twist result $\sigma_{\mathrm{L}}^{(2)} \simeq$ $\sigma_{0} \sum_{f} e_{f}^{2} \frac{\alpha_{\mathrm{em}}}{\pi} \xi$.

The longitudinal twist- 4 and twist- 8 terms give sizeable negative corrections to the leading-twist contribution, mainly at $\xi=\frac{Q_{\mathrm{s}}^{2}}{Q^{2}} \approx 1$. It is expected that precise low $Q^{2}$ measurements of $F_{\mathrm{L}}$ at small- $x$ could reveal this important feature. This is investigated in the analysis presented here, considering the recent $\mathrm{H} 1$ preliminary data on the longitudinal structure function. It should be noticed that although $F_{2}$ had been measured in this region with accurate precision, its longitudinal and transverse twist-4 contributions have opposite signs and almost the same order of magnitude. Hence, they approximately cancel each 
other and produce either a small twist- 4 correction. Therefore, for the inclusive $F_{2}$ structure function the highertwist corrections are hidden in the mismatch between the longitudinal and transverse higher-twist corrections.

Despite the saturation model being very successful in describing HERA data, its functional form is only an approximation of the theoretical non-linear QCD approaches. On the other hand, an analytical expression for the dipole cross section can be obtained within the BFKL formalism. Currently, intense theoretical studies are being performed towards an understanding of the BFKL approach in the border of the saturation region $[44,45]$. In particular, the dipole cross section has been calculated in both LO and NLO BFKL approach in the geometric scaling region [46]. It reads

$\sigma_{\mathrm{dip}}(x, \boldsymbol{r})=\sigma_{0}\left[\boldsymbol{r}^{2} Q_{\mathrm{sat}}^{2}(x)\right]^{\gamma_{\mathrm{sat}}} \exp \left[-\frac{\ln ^{2}\left(\boldsymbol{r}^{2} Q_{\mathrm{sat}}^{2}\right)}{2 \beta \bar{\alpha}_{\mathrm{s}} Y}\right]$,

where $\sigma_{0}=2 \pi R_{p}^{2}\left(R_{p}\right.$ is the proton radius) is the overall normalization and the power $\gamma_{\text {sat }}$ is the (BFKL) saddle point in the vicinity of the saturation line $Q^{2}=Q_{\text {sat }}^{2}(x)$. In addition, the anomalous dimension is defined as $\gamma=1-$ $\gamma_{\text {sat }}$. As usual in the BFKL formalism, $\bar{\alpha}_{\mathrm{s}}=N_{c} \alpha_{\mathrm{s}} / \pi, \beta \simeq$ $28 \zeta(3)$ and $Y=\ln (1 / x)$. The quadratic diffusion factor in the exponential gives rise to the scaling violations.

The dipole cross section in (8) does not include an extrapolation from the geometric scaling region to the saturation region. This has been recently implemented in [21], where the dipole amplitude $\mathcal{N}(x, \boldsymbol{r})=\sigma_{\text {dip }} / 2 \pi R_{p}^{2}$ was constructed to smoothly interpolate between the limiting behaviors analytically under control: the solution of the BFKL equation for small dipole sizes, $\boldsymbol{r} \ll 1 / Q_{\text {sat }}(x)$, and the Levin-Tuchin law [47] for larger ones, $\boldsymbol{r} \gg 1 / Q_{\text {sat }}(x)$. A fit to the structure function $F_{2}\left(x, Q^{2}\right)$ was performed in the kinematical range of interest, showing that it is not very sensitive to the details of the interpolation (for a comprehensive phenomenological analysis of the HERA results using the numerical solution of the BK equation see [48]). The dipole cross section was parametrized as follows:

$$
\begin{aligned}
& \sigma_{\mathrm{dip}}(x, \boldsymbol{r}) \\
& =\sigma_{0} \begin{cases}\mathcal{N}_{0}\left(\frac{\boldsymbol{r} Q_{\mathrm{sat}}}{2}\right)^{2\left(\gamma_{\mathrm{sat}}+\frac{\ln \left(2 / \boldsymbol{r}_{\left.Q_{\mathrm{sat}}\right)}\right.}{\kappa \lambda Y}\right)}, & \text { for } \boldsymbol{r} Q_{\mathrm{sat}}(x) \leq 2, \\
1-\exp ^{-a \ln ^{2}\left(b \boldsymbol{r} Q_{\mathrm{sat}}\right)}, & \text { for } \boldsymbol{r} Q_{\mathrm{sat}}(x)>2,\end{cases}
\end{aligned}
$$

where the expression for $\boldsymbol{r} Q_{\text {sat }}(x)>2$ (saturation region) has the correct functional form, as obtained either by solving the Balitsky-Kovchegov (BK) equation $[5,6]$, or from the theory of the color glass condensate (CGC) [25]. Hereafter, we label the model above by CGC. The coefficients $a$ and $b$ are determined from the continuity conditions of the dipole cross section at $r Q_{\text {sat }}(x)=2$. The coefficients $\gamma_{\text {sat }}=0.63$ and $\kappa=9.9$ are fixed from their LO BFKL values. In our further calculations will be used the parameters $R_{p}=0.641 \mathrm{fm}, \lambda=0.253, x_{0}=0.267 \times 10^{-4}$ and
$\mathcal{N}_{0}=0.7$, which give the best fit result. A large $x$ threshold factor $(1-x)^{5}$ will be also considered, for the sake of completeness.

Recently, this model has also been used in phenomenological studies of the vector meson production [22] and the diffractive processes [23] at HERA as well as hadron production in nuclear collisions at RHIC [24]. Here we compare for the first time this model with the recent $\mathrm{H} 1$ data for the longitudinal structure function.

\section{Results and discussions}

Let us present the numerical results coming out of the saturation models (GBW and CGC) and from the usual collinear approach. In Figs. 1 and 2 the predictions of various theoretical models are compared with the $\mathrm{H} 1$ experimental results for the longitudinal structure function $[28,50]$. In particular, in Fig. 1 we compare the distinct predictions with the recent $\mathrm{H} 1$ preliminary $F_{\mathrm{L}}$ data at $Q^{2}=0.75,1.35 \mathrm{GeV}^{2}$, as determined from the 1999 minimum bias and the 2000 shifted vertex H1 data. These points were extracted from the plots on [28]. The data show that $F_{\mathrm{L}}$ remains non-zero down to the lowest $Q^{2}$ values measured and already distinguish between the different models in the low- $x$ region. The previous $F_{\mathrm{L}}$ data [50] at larger $Q^{2}$ are also presented.

In Fig. 1, the lowest $Q^{2}$ bins are shown, in particular the new $Q^{2}=0.75$ and $Q^{2}=1.35 \mathrm{GeV}^{2}$ measurements. At this region, the two saturation models (GBW and CGC) give very similar results; as a consequence they have a similar behavior in the transition to the saturation regime. Namely, their main differences are in the large virtuality region, where CGC depends on the BFKL anomalous dimension at the saturation vicinity as referred to before. The data description is very consistent, mostly at the lowest $Q^{2}$ points where the usual collinear approaches are unable to produce reliable results. For the sake of comparison, a leading-twist calculation is also presented. We have used (2) and considered three different choices for the parton distributions. The predictions from the GRV98 parameterization, which is obtained using the DGLAP evolution equation, and the EHKQS gluon function [49], which contains corrections from the non-linear GLR evolution equation, are not in agreement with experimental data, even at large $Q^{2}$. The EHKQS gluon distribution slows down the dependence on $x$, but it is not enough either to reach the upper limit of error bars. Notice that at $Q^{2}=0.75 \mathrm{GeV}^{2}$ a DGLAP approach is unable to give reliable results, though a backward QCD evolution is possible. For the GRV98 case, we have extrapolated the $Q^{2}=0.85 \mathrm{GeV}^{2}$ initial condition down to $Q^{2}=0.75 \mathrm{GeV}^{2}$ once they are very closer. Similarly, for $Q^{2}=1.35 \mathrm{GeV}^{2}$, we present the EHKQS prediction for $Q^{2}=1.4 \mathrm{GeV}^{2}$ which is the lowest available $Q^{2}$ for this parameterization. On the other hand, the predictions for $F_{\mathrm{L}}$ using the MRST parameterization reasonably describe the H1 data, which is directly associated to the behavior assumed for the parton distributions in the initial evolution scale $Q^{2}=1.0 \mathrm{GeV}^{2}$. In this case, the behavior of the sea distribution is independent of the gluon 

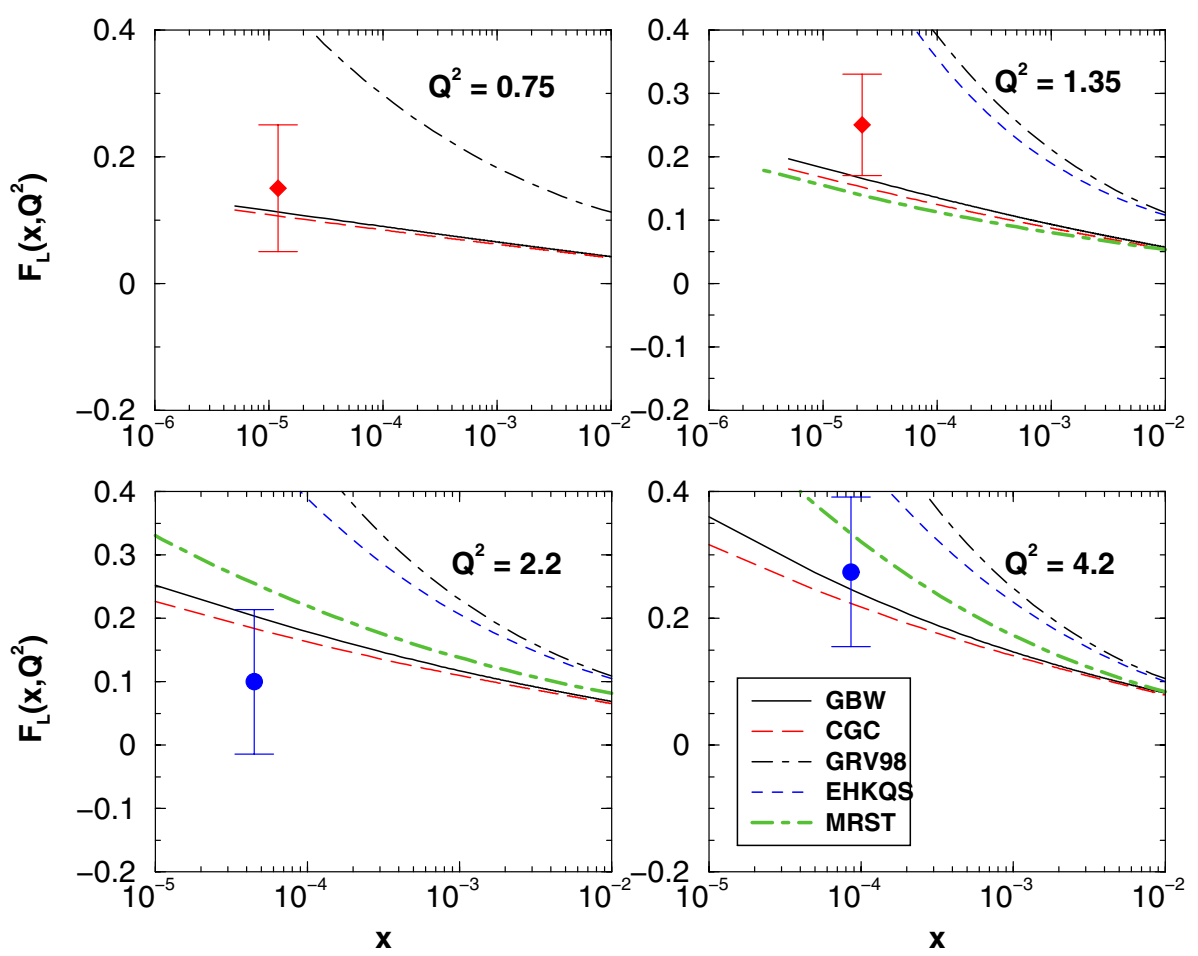

Fig. 1. The results for $F_{\mathrm{L}}\left(x, Q^{2}\right)$ as a function of $x$ at fixed low $Q^{2}$ values. The numerical results for the saturation models (GBW and CGC) as well as leading-twist DGLAP approach for three inputs for the gluon distribution (GRV98, EHKQS and MRST) are presented. Data are from from the H1 Collaboration

one, with the input gluon distribution being valence-like $\left(x g \propto x^{0.10}\right)$, while the input sea distribution has a steep growth at small $x\left(x S \propto x^{-0.19}\right)$.

In Fig. 2 one presents the high $Q^{2}$ analysis. Once again, the saturation models give a reasonable description of the data, producing a milder $x$ growth than the DGLAP results. Their overall normalization also is smaller than the
DGLAP analysis, becoming closer as virtuality increases. When comparing the DGLAP results, one verifies that the intermediate $Q^{2}$ region at small $x$ is an adequate kinematical region to study non-linear QCD corrections to the gluon distribution. There, very precise measuments of $F_{\mathrm{L}}$ or large statistics could constrain the size of those corrections.
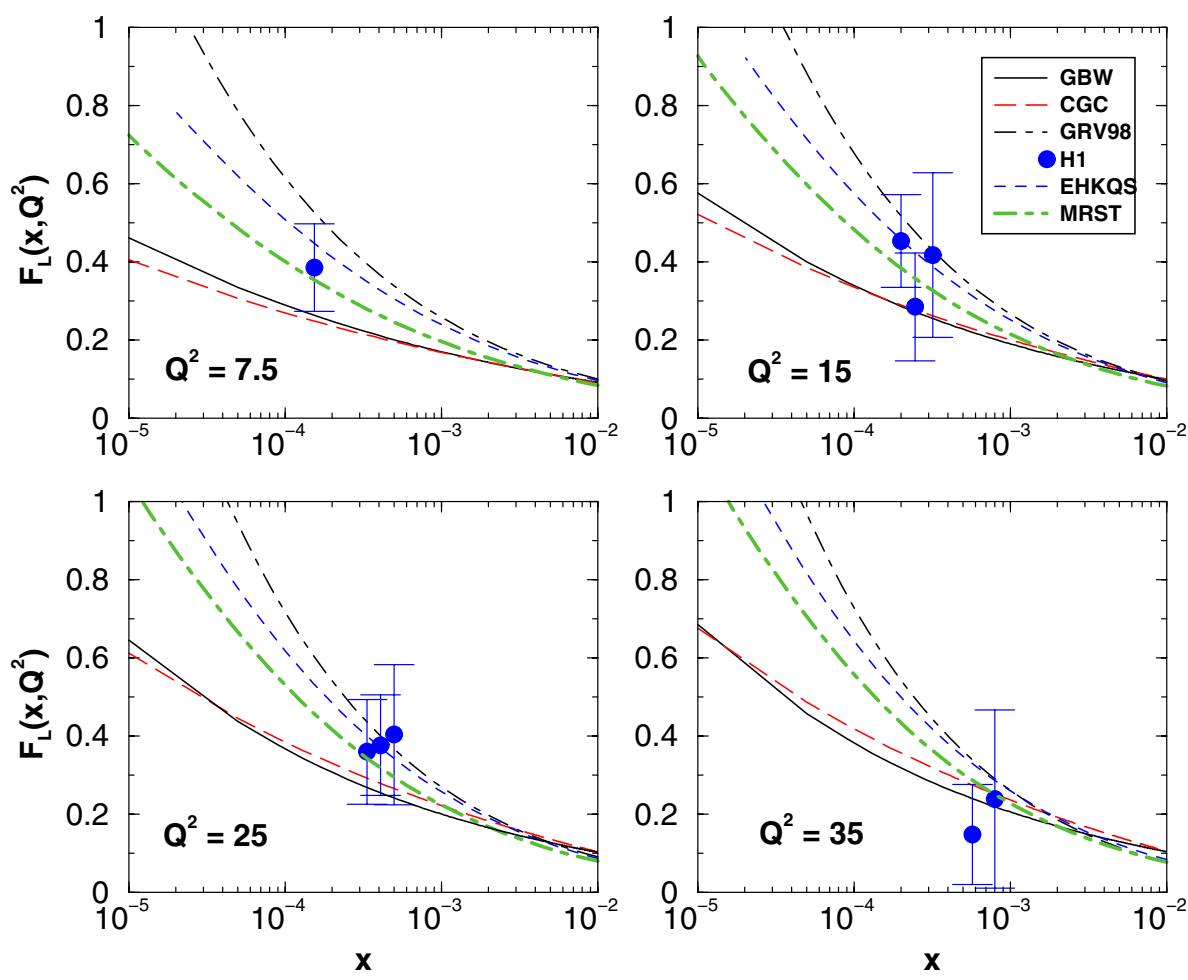

Fig. 2. The results for $F_{\mathrm{L}}\left(x, Q^{2}\right)$ as a function of $x$ at fixed large $Q^{2}$ values. Same notation as in previous figure 

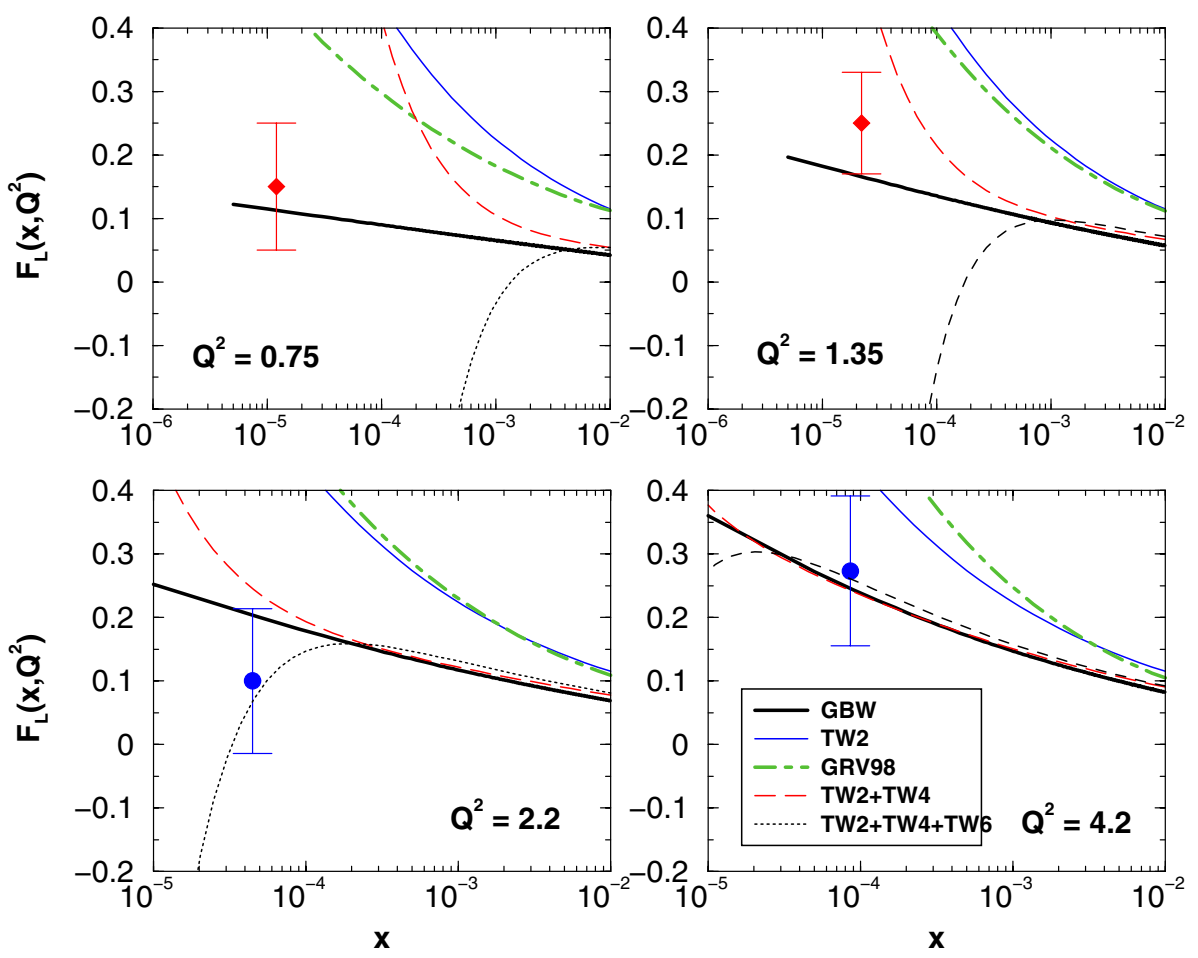

Fig. 3. The results for $F_{\mathrm{L}}\left(x, Q^{2}\right)$ as a function of $x$ at fixed large $Q^{2}$ values for the saturation model (GBW) and the sum of its twist contributions. The leading-twist (twist-2) and the first two higher-twist (twist-4 and twist-6) corrections are presented. The leadingtwist DGLAP results (using GRV98 gluon pdf) is shown for comparison

Finally, in Fig. 3 we present a comparison among the contributions of the different twists. In order to do this, we show separately the result from the summation of the different twists for the analytically simple expression in (5) and (6) for the saturation model. For comparison we also present the GBW prediction, which represents the full higher-twist resummation. In order to compare the present analysis with a leading-order and leading-twist calculation, we present in addition the results of (2) considering the GRV98 gluon distribution. We can see that the twist2 contribution from the saturation model is in complete numerical agreement with the DGLAP result, showing a consistent reproduction of leading-twist contribution in its twist resummation. We see that the twist-4 and twist6 terms give important contributions, strongly modifying the magnitude of the longitudinal structure function and its $x$-behavior. Moreover, the $x$ value where the curves become distinct is $Q^{2}$-dependent, as expected from the energy dependence present in the saturation scale and consequently in the variable $\xi$. In particular, for $Q^{2}=4.2 \mathrm{GeV}^{2}$ we see that the sum of the twist- 2 and twist- 4 terms reasonable reproduces the GBW prediction for $x>10^{-5}$, while for $Q^{2}=2.2 \mathrm{GeV}^{2}$ this approximation is only valid for $x>10^{-4}$. For smaller values of $Q^{2}$ only the full resummation gives a good description of the experimental data, which demonstrate that a twist summation term by term (i.e., summing the first contributing terms) would either be incomplete in that kinematical regime.

As a summary, we have analyzed the longitudinal structure function at low $Q^{2}$ and small- $x$, which directly depends on the gluon distribution function, within the saturation approach. In particular, we have shown that the saturation models (GBW and CGC) consistently describe the recent low $Q^{2}$ H1 data, even at very low virtualities.
Moreover, we have presented the higher-twist contributions, using the simple analytical expressions provided by the GBW model. It is verified that they play an important role at small- $x$ for the virtualities considered here. Concerning the leading twist DGLAP analysis, which we have considered for the sake of comparison, it is shown that non-linear GLR corrections to the gluon distribution function are not enough to bring the numerical analysis to the recent experimental results. However, we have shown that the intermediate $Q^{2}$ region should be an important kinematical region where the size of such corrections could be investigated. In general, $F_{\mathrm{L}}$ is an outstanding observable testing both parton saturation and twist resummation. Therefore, more precise data and/or more statistics are increasingly desirable at low $Q^{2}$ and small $x$. Significant further progress in $F_{\mathrm{L}}$ measurements at HERA can only be made by reducing the proton beam energy. A run with reduced proton beam energies is planned for the next few years.

Acknowledgements. M.V.T.M. thanks for the support of the High Energy Physics Phenomenology Group, GFPAE IFUFRGS, Brazil. This work was partially financed by the Brazilian funding agencies CNPq and FAPERGS.

\section{References}

1. L.V. Gribov, E.M. Levin, M.G. Ryskin, Phys. Rept. 100, 1 (1983)

2. A.H. Mueller, J. w. Qiu, Nucl. Phys. B 268, 427 (1986)

3. A.L. Ayala, M.B. Gay Ducati, E.M. Levin, Nucl. Phys. B 493, 305 (1997); Nucl. Phys. B 511, 355 (1998) 
4. L.D. McLerran, R. Venugopalan, Phys. Rev. D 49, 2233 (1994)

5. I. Balitsky, Nucl. Phys. B 463, 99 (1996)

6. Y.V. Kovchegov, Phys. Rev. D 60, 034008 (1999); Phys. Rev. D 61, 074018 (2000)

7. E. Iancu, A. Leonidov, L. McLerran, Nucl. Phys. A 692, 583 (2001); E. Ferreiro, E. Iancu, A. Leonidov, L. McLerran, Nucl. Phys. A 703, 489 (2002)

8. A.L. Ayala Filho, M.B. Gay Ducati, E.M. Levin, Eur. Phys. J. C 8, 115 (1999)

9. A.L. Ayala Filho, M.B. Gay Ducati, V.P. Goncalves, Phys. Rev. D 59, 054010 (1999)

10. K. Golec-Biernat, M. Wüsthoff, Phys. Rev. D 59, 014017 (1999); Phys. Rev. D 60, 114023 (1999)

11. J. Bartels, K. Golec-Biernat, H. Kowalski, Phys. Rev D 66, 014001 (2002)

12. H. Kowalski, D. Teaney, Phys. Rev. D 68, 114005 (2003)

13. C. Marquet, R. Peschanski, Phys. Lett. B 587, 201 (2004)

14. A.M. Stasto, K. Golec-Biernat, J. Kwiecinski, Phys. Rev. Lett. 86, 596 (2001)

15. A.C. Caldwell, M.S. Soares, Nucl. Phys. A 696, 125 (2001)

16. N. Timneanu, J. Kwiecinski, L. Motyka, Eur. Phys. J. C 23, 513 (2002)

17. C. Brenner Mariotto, M.B. Gay Ducati, M.V.T. Machado, Phys. Rev. D 66, 114013 (2002)

18. L. Favart, M.V.T. Machado, Eur. Phys. J. C 29, 365 (2003); C 34, 429 (2004)

19. J.R. Forshaw, G. Kerley, G. Shaw, Phys. Rev. D 60, 074012 (1999); M. McDermott, L. Frankfurt, V. Guzey, M. Strikman, Eur. Phys. J. C 16, 641 (2000); E. Gotsman et al., J. Phys. G 27, 2297 (2001); A. Donnachie, H.G. Dosch, Phys. Rev. D 65, 014019 (2002); M.B. Gay Ducati, M.V.T. Machado, Phys. Rev. D 65, 114019 (2002); M.A. Betemps, M.B. Gay Ducati, M.V.T. Machado, Phys. Rev. D 66, 014018 (2002)

20. V.P. Goncalves, M.V.T. Machado, Phys. Rev. Lett. 91, $202002(2003)$

21. E. Iancu, K. Itakura, S. Munier, Phys. Lett. B 590, 199 (2004)

22. J.R. Forshaw, R. Sandapen, G. Shaw, Phys. Rev. D 69, 094013 (2004)

23. J.R. Forshaw, R. Sandapen, G. Shaw, Phys. Lett. B 594, $283(2004)$

24. J. Jalilian-Marian, Nucl. Phys. A 739, 319 (2004); nuclth/0402080

25. E. Iancu, R. Venugopalan, hep-ph/0303204
26. J. Bartels, K. Golec-Biernat, K. Peters, Eur. Phys. J. C 17, 121 (2000)

27. E. Gotsman, E. Levin, U. Maor, L.D. McLerran, K. Tuchin, Nucl. Phys. A 683, 383 (2001); Phys. Lett. B 506, 289 (2001)

28. E.M. Lobodzinska, Acta Phys. Polon. B 35, 223 (2004); hep-ph/0311180

29. L. Favart et al., Z. Phys. C 72, 425 (1996)

30. R.S. Thorne, Phys. Lett. B 418, 371 (1998)

31. B. Badelek, J. Kwiecinski, A. Stasto, Z. Phys. C 74, 297 (1997)

32. A.V. Kotikov, A.V. Lipatov, N.P. Zotov, Eur. Phys. J. C 27, 219 (2003); hep-ph/0403135

33. A.Y. Illarionov, A.V. Kotikov, G. Parente Bermudez, hep$\mathrm{ph} / 0402173$

34. A.D. Martin, R.G. Roberts, W.J. Stirling, R.S. Thorne, Phys. Lett. B 531, 216 (2002); Eur. Phys. J. C 23, 73 (2002)

35. J. Pumplin, D.R. Stump, J. Huston, H.L. Lai, P. Nadolsky, W.K. Tung, JHEP 0207, 012 (2002)

36. R.S. Thorne, Int. J. Mod. Phys. A 19, 1074 (2004) [hep$\mathrm{ph} / 0309343]$

37. R.S. Thorne, Phys. Rev. D 64, 074005 (2001)

38. S. Alekhin, Phys. Rev. D 68, 014002 (2003); D 63, 094022 (2001)

39. A.D. Martin, R.G. Roberts, W.J. Stirling, R.S. Thorne, Phys. Lett. B 443, 301 (1998)

40. G. Altarelli, G. Martinelli, Phys. Lett. B 76, 89 (1978)

41. A.M. Cooper-Sarkar, G. Ingelman, K.R. Long, R.G. Roberts, D.H. Saxon, Z. Phys. C 39, 281 (1988)

42. M. Gluck, E. Reya, A. Vogt, Eur. Phys. J. C 5, 461 (1998)

43. N.N. Nikolaev, B.G. Zakharov, Z. Phys. C 49, 607 (1991); Z. Phys. C 53, 331 (1992); A.H. Mueller, Nucl. Phys. B 415, 373 (1994); A.H. Mueller, B. Patel, Nucl. Phys. B 425, 471 (1994)

44. E. Iancu, K. Itakura, L. McLerran, Nucl. Phys. A 708, 327 (2002)

45. S. Munier, S. Wallon, Eur. Phys. J. C 30, 359 (2003)

46. A.H. Mueller, D.N. Triantafyllopoulos, Nucl. Phys. B 640, 331 (2002); D.N. Triantafyllopoulos, Nucl. Phys. B 648, 293 (2003); A.H. Mueller, Nucl. Phys. A 724, 223 (2003)

47. E. Levin, K. Tuchin, Nucl. Phys. B 573, 833 (2000)

48. E. Gotsman, E. Levin, M. Lublinsky, U. Maor, Eur. Phys. J. C 27, 411 (2003)

49. K.J. Eskola, H. Honkanen, V.J. Kolhinen, J. w. Qiu, C.A. Salgado, Nucl. Phys. B 660, 211 (2003)

50. C. Adloff et al. [H1 Collaboration], Eur. Phys. J. C 21, 33 (2001) 